\title{
Caracterización nutricional y funcional de la harina de
}

\section{mashua}

\section{Nutritional and functional importance of mashua flour (Tropaeolum tuberosum)}

\author{
María Verónica González Cabrera ${ }^{1}$, Georgina Ipatia Moreno Andrade $^{2}$, \& Sandra \\ Elizabeth López Sampedro ${ }^{3}$
}

DOI: https://doi.org/10.33262/concienciadigital.v3i3.1299

\begin{abstract}
.
The present investigation proposes a characterization of mashua flour (Tropaeolum tuberosum) from the nutritional and functional point of view to establish the utility of its application in the elaboration of sausages. It began with obtaining the flour with a process of selection, cleaning, washing and cutting of the fresh mashua. After a drying, milling and sieving process, the samples were analyzed taking into account the methods established by the Ecuadorian Technical Standards INEN and the Official Methods of Analysis of the Association (AOAC). The proximal analysis performed on the mashua flour samples reflect an average moisture content of $10.46 \pm 0.09 \%$, the protein contribution is in a range of $12.34 \pm 0.08 \%$, while the content ash has an average value of $4.66 \pm 0.10 \%$. Regarding the fat content, an average result of $0.81 \pm 0.24 \%$ was obtained and with respect to the fiber an average of $7.07 \pm 0.009 \%$. For carbohydrates the average result was $64.67 \pm 0.14 \%$. From the analysis of the CRA water holding capacity, a percentage was obtained that varies between 5.05 and $8.63 \%$ for a temperature range of 50 to $80^{\circ} \mathrm{C}$, demonstrating with this result that the temperature has a significant impact on the CRA. The samples of mashua flour analyzed are within the established parameters and are of acceptable physical-chemical and functional quality.
\end{abstract}

\footnotetext{
${ }^{1}$ Escuela Superior Politécnica de Chimborazo, Facultad de Ciencias Pecuarias. Riobamba, Ecuador. mariav.gonzalez@espoch.edu.ec

${ }^{2}$ Escuela Superior Politécnica de Chimborazo, Facultad de Ciencias Pecuarias. Riobamba, Ecuador.georgina.moreno@espoch.edu.ec

${ }^{3}$ Escuela Superior Politécnica de Chimborazo, Facultad de Ciencias Pecuarias. Riobamba, Ecuador. sandra.lopez@espoch.edu.ec
} 
Keywords: Physical chemical analysis, proximal composition, functional analysis, mashua, flour.

\section{Resumen.}

La presente investigación propone una caracterización de la harina de mashua (Tropaeolum tuberosum) desde el punto de vista nutricional y funcional para establecer la utilidad de su aplicación. Se inició con la obtención de la harina luego de un proceso de selección, limpieza, lavado y troceado, secado, molienda y tamizado se procedió a analizar las muestras tomando en cuenta los métodos establecidos por las Normas Técnicas Ecuatorianas INEN y la AOAC (Official Methods of Analysis of the Association). El análisis proximal realizado a las muestras de harina de mashua reflejan un contenido de humedad promedio de $10,46 \pm 0,09 \%$, el aporte de proteína se encuentra en un rango de $12,34 \pm 0,08 \%$, mientras que el contenido de cenizas presenta un valor promedio de $4,66 \pm 0,10 \%$. En cuanto al contenido de grasa se obtuvo un resultado promedio de $0,81 \pm 0,24 \%$ y con respecto a la fibra un promedio de $7,07 \pm 0,009 \%$. Para carbohidratos el resultado promedio fue de $64,67 \pm 0,14 \%$. Del análisis funcional de la harina de mashua se obtiene un valor de $\mathrm{pH}$ igual a 5,5 $\pm 0,02$ y un porcentaje de acidez de $1,08 \pm 0,025 \%$, además la capacidad de retención de agua CRA mostró un porcentaje que varía entre 5,05 y $8,63 \%$ para un rango de temperaturas de 50 a $80^{\circ} \mathrm{C}$, demostrándose con este resultado que la temperatura incide significativamente en la CRA. Las muestras de harina de mashua analizadas cumplen con los parámetros establecidos y son de calidad físico-química y funcional aceptable.

Palabras clave: Análisis físico químico, composición proximal, análisis funcional, mashua, harina.

\section{Introducción.}

Una de las maneras de promover el aprovechamiento industrial de las raíces y tubérculos andinos depende en su mayoría de disponer de información acerca de la composición química, física, nutricional y funcional, la cuantificación de estos parámetros permitirá conocer su aplicación y utilidad.

El Ecuador en su región andina concentra una de las zonas más favorables para el cultivo y producción de este tipo de raíces y tubérculos. Según Grau, "la mashua es considerada como el tubérculo más promisorio debido a la rusticidad del cultivo, la tolerancia a plagas y su alta productividad ya que su gran rendimiento supera a los demás tubérculos incluyendo la papa, con bajos niveles de insumos; además posee una amplia gama de fotoquímicos que no solo protegen al mismo cultivo sino también contra las plagas de otros cultivos". (Grau, 2003)

Determinar la composición química y por ende del valor nutricional; así como el rendimiento de las raíces de tubérculos andinos permiten establecer usos y aplicaciones 
por ejemplo en dietas de animales de interés económico en áreas como la ganadería y la avicultura. Ruiz y Pavón manifiestan que la mashua, "Tropaeolium tuberosum” pertenece a la familia de las "Tropaeolaceae", la cual incluye 86 especies ampliamente distribuidas. (Arbizu, 1993)

La mashua es un tubérculo comestible originario de los Andes se cultiva principalmente en Perú y Bolivia, así como en Ecuador en la sierra, principalmente en las provincias de Bolívar, Pichincha, Cotopaxi y Tungurahua, en parcelas de consumo familiar. Un estudio en el año 2002 del Instituto Nacional Autónomo de Investigaciones Agropecuarias, refleja que "la demanda de este tubérculo en el Ecuador decayó pese a su gran valor nutritivo; sin embargo, se cultiva entre los 2.000 y 4.000 metros de altura, con rendimientos potenciales de 30 toneladas por hectárea." (Monteros A, 2012); constituye una buena fuente de alimentación para más de 9 millones de personas que viven en la Cordillera de los Andes. De hecho, se dice que la mashua ocupa el cuarto lugar, después de la papa, entre los tubérculos más importantes de las regiones andinas.

Este tubérculo suele ser de color blanco amarillento, pero también en el Ecuador existen más de 100 variedades de mashua, entre las que se mencionan "Amarilla tardía o Quilla zapallo, Amarilla chaucha, Pulsito o Puzungo, Blanca, Rodilla de Jesucristo y Morada" (Espinoza, 2002). "Su sabor es más suave que el de la papa, con un regusto floral y picante, se sabe que el tratamiento post-cosecha tradicional al que son sometidos los tubérculos de mashua antes de consumirlos es el soleado, el cual se aplica con el objetivo de eliminar el sabor amargo e incrementar el dulzor". (Grau, 2003)

El interés en la mashua se ha incrementado debido a que se ha demostrado que tiene propiedades nutricionales y funcionales, esto hace referencia a que "posee un alto contenido de compuestos fenólicos, carotenoides y antocianinas, además de una elevada capacidad antioxidante en comparación con otros tubérculos como la papa, oca y olluco" (Campos, y otros, 2006)

Además, los tubérculos de la mashua son ricos en agua, en carbohidratos, proteínas y nutrientes como la vitamina $C$; el contenido de vitamina $C$ en 100 gramos de mashua representa más del doble de una naranja. "Es nutritivo y bajo en calorías; contiene grandes cantidades de glucosinolatos e isotiocianatos, compuestos reconocidos por sus propiedades antibióticas, diuréticas y anticancerígenas". (Cultivariable, 2018)

Fairlie, Morales y Holle (1999) mencionan que, eliminando el agua presente en las raíces y tubérculos andinos, se produce una concentración de sus nutrientes, motivo por el cual su potencial nutritivo se incrementa, de igual manera estas harinas resultado de la deshidratación no se destinan al consumo directo sino a su procesamiento". (citado en Bernabé y Cancho, 2017)

En este sentido el objetivo de la investigación es determinar las características nutricionales y funcionales de la mashua para uso industrial, principalmente en la 
elaboración de productos o derivados cárnicos para su aprovechamiento en la industria alimentaria gracias a su gran potencial de transformación en harinas, ya que se dispone de información sobre sus características fisicoquímicas como el tamaño de partícula, ph, acidez y densidad que indican el buen estado de conservación y el grado de finura, respectivamente.

Finalmente, las propiedades funcionales de este tubérculo andino, tales como: la capacidad de retención de agua depende de los puentes de hidrógeno que forman las proteínas con el agua; así mismo, la capacidad de retención de aceite favorece la conservación del aroma con lo cual se incrementa el nivel de palatabilidad, de manera similar la característica hidrofóbica de las proteínas es fundamental en el proceso de absorción de grasa. (Bernabé y Cancho, 2017). Las proteínas de estos tubérculos andinos presentan capacidad de hinchamiento que se puede considerar como una propiedad funcional ya que es indispensable para la elaboración de alimentos viscosos y para ingresar al desarrollo de nuevos productos como en repostería, panadería, pastas, sopas, fideos, salsas, masas y otros productos horneados

\section{Metodología.}

\section{Material vegetal.}

Selección, limpieza y desinfección: La mashua fue adquirida de diferentes procedencias, posteriormente se seleccionan las que no presentaron daños mecánicos y/o evidencias de ataques de plagas o enfermedades, es decir las muestras de tubérculos con buena calidad fitosanitaria. De manera subsiguiente a la selección, se procede al lavado por sumersión en agua, así como un proceso de cepillado para eliminar residuos de tierra o materia orgánica que puedan estar adheridas a la cáscara del tubérculo y así facilitar su desinfección en la etapa siguiente.

Para la desinfección las muestras de tubérculos fueron sometidas a inmersión por 5 minutos en una solución hipoclorito de sodio (100ppm) con el objetivo de eliminar cualquier agente patógeno presente.

Troceado y secado: La materia prima se peló en forma manual y se partió en rodajas de aproximadamente $1 \mathrm{~cm}$ de ancho, luego cada rodaja fue cortada en cuatro partes iguales y se colectaron en bandejas de aluminio que se ubicaron en un horno de secado a una temperatura de $65^{\circ} \mathrm{C}$ durante 18 horas hasta peso constante. Una vez seco se transfirió a un molino con un tamaño de partícula menor a $850 \mathrm{~m} \mathrm{~m}$ hasta obtener una harina, para luego tamizar y eliminar partículas extrañas

\section{Determinación de pérdida por calentamiento (humedad).}

Para las muestras de harina de mashua se aplicó el método señalado en la norma técnica ecuatoriana NTE INEN 518 Harina de origen vegetal. Determinación de pérdida por calentamiento. Este método se basa en calentar la muestra de harina a $130 \pm 3^{\circ} \mathrm{C}$ durante 
30 minutos, enfriar en el desecador las muestras hasta temperatura ambiente y pesar con una aproximación de $0,1 \mathrm{mg}$. Se repiten las operaciones de calentamiento, enfriamiento y pesaje hasta que la diferencia entre los resultados de dos operaciones no supere $0,1 \mathrm{mg}$. (INEN, 1980)

\section{Contenido de cenizas totales.}

Se cuantificó el contenido de cenizas totales, siguiendo los métodos de referencia (AOAC, 2005). Las muestras (0,5-1 g) fueron sometidas a deshidratación en estufa a $105^{\circ} \mathrm{C}$ hasta alcanzar peso constante. Luego, se dejaron enfriar en un desecador y se pesaron. Los resultados finales se expresaron como porcentaje (\%) con relación al peso inicial de la muestra. (INEN, 1980)

\section{Cuantificación de proteína cruda.}

Para este ensayo se aplicó el método contemplado en la norma técnica ecuatoriana NTE INEN 519 Harinas de origen vegetal. Determinación de proteína. Se cuantificó el nitrógeno total de las muestras $(1 \mathrm{~g})$ mediante el método Kjeldahl. Se utilizó una unidad de digestión y una unidad de destilación. El contenido de proteína cruda se calculó usando el factor de conversión 6,25. Y los resultados se expresaron como porcentaje (\%) sobre base seca $\left(\mathrm{a} 65^{\circ} \mathrm{C}\right)$. (INEN, 1980)

\section{Extracto etéreo.}

Las muestras ( $1 \mathrm{~g}$ ) se extrajeron con hexano durante $4 \mathrm{~h}$ en un equipo Goldfish. El residuo obtenido en el beaker de extracción luego de la recuperación y evaporación del solvente (grasa cruda) fue cuantificado gravimétricamente. Los resultados se expresaron como porcentaje $(\%)$ sobre base seca $\left(\mathrm{a} 65^{\circ} \mathrm{C}\right)$.

\section{Determinación de Fibra Cruda.}

Este ensayo fue realizado siguiendo el método presentado en la NTE-INEN 522:2013. Harinas de origen vegetal. Determinación de la Fibra cruda, el mismo que consiste en digerir la muestra sin grasa con solución de ácido sulfúrico, lavar y nuevamente digerir con solución de hidróxido de sodio, lavar, secar y pesar. Calcinar hasta destrucción de la materia orgánica. La pérdida de peso después de la calcinación es el contenido de fibra cruda en la muestra (INEN, 2013).

Análisis físico-químicos: Las muestras de harina se almacenaron en recipientes plásticos a temperatura ambiente en el laboratorio de Nutrición y Bromatología de la Facultad de Ciencias Pecuarias a fin de realizar los análisis bromatológicos y microbiológicos correspondientes según (AOAC 2005).

\section{Análisis funcional.}




\section{Determinación de pH.}

Este ensayo se realizó con base en la NTE INEN 586 Determinación de la concentración de Ión hidrógeno o $\mathrm{pH}$ en harinas de origen vegetal. El equipo utilizado en el ensayo fue un potenciómetro, con electrodos de vidrio estandarizado. Se toma una muestra representativa de la harina evitando su exposición al aire mucho tiempo. Se procede a pesar $10 \mathrm{~g} \pm 0,1 \mathrm{~g}$ de muestra colocándola en el vaso de precipitación, se añaden $100 \mathrm{~cm} 3$ de agua destilada y se homogeniza la solución durante $30 \mathrm{~min}$ a $25^{\circ} \mathrm{C}$, se deja en reposo durante $10 \mathrm{~min}$ hasta que el líquido decante. Se decanta el sobrenadante en el vaso de precipitación y se procede a la determinación del $\mathrm{pH}$ introduciendo el potenciómetro en la solución de líquido sobrenadante. (INEN, 2013).

\section{Determinación de acidez titulable.}

El método para determinar el contenido de acidez titulable es el descrito en la NTE INEN 5212013 para harinas de origen vegetal, expresándose como ácido sulfúrico. Se titula la acidez como una solución estandarizada de hidróxido de sodio $0,02 \mathrm{~N}$, usando fenolftaleína como indicador. De manera inicial se toman muestras representativas de la harina de mashua que son colocadas en recipientes herméticos, limpios y secos, evitando exponerlas al aire mucho tiempo. Una vez homogenizadas se pesan $5 \mathrm{~g}$ de las muestras y se transfirieren a un matraz Erlenmeyer de $100 \mathrm{~cm} 3$. Se procede a agregar lentamente 50 $\mathrm{cm} 3$ de alcohol de 90\% (V/V) neutralizado, con el matraz Erlenmeyer tapado se agita fuertemente. Posteriormente se deja en reposo durante 24 horas, agitando de vez en cuando. Una vez transcurrido este tiempo se toman alícuotas de $10 \mathrm{~cm} 3$ del líquido claro sobrenadante y se ubican en el matraz Erlenmeyer al que se le agregan $2 \mathrm{~cm} 3 \mathrm{de}$ fenolftaleína. Se procede a la titulación con una solución 0,02 N de hidróxido de sodio, agregándola gota a gota y con agitación constante hasta conseguir que el color rosado persista durante $30 \mathrm{~s}$. Se procede a leer en la bureta el volumen de solución empleada, con aproximación a $0,05 \mathrm{~cm} 3$ (INEN, 2013).

\section{Determinación de la Actividad de agua aw:}

Esta determinación permite definir condiciones de conservación y almacenamiento para las muestras de harina de mashua. El dispositivo utilizado corresponde al equipo Aw Sprint, de la marca Novasina, modelo TH-500. Este equipo tiene una celda con un sensor a través del cual se determina la humedad relativa de la muestra. El Aw Sprint funciona entre $0^{\circ} \mathrm{C}$ a $50^{\circ} \mathrm{C}$. Para la medición se coloca una muestra de la harina de mashua en la celda y se realiza la lectura a $25^{\circ} \mathrm{C}$, por ser la temperatura reportada en datos de bibliografía que se usan como referencia.

\section{Determinación de la Capacidad de Retención de agua.}

Para esta determinación se prepararon disoluciones al 1\% (p/v) de harina de mashua en agua. Para establecer las condiciones de temperatura se ubicó en baño maría estas disoluciones a 50,60,70 y $80^{\circ} \mathrm{C}$ por un tiempo de 15 minutos con agitación constante. 
Posteriormente las muestras fueron centrifugadas a $1000 \mathrm{rpm}$ por 10 minutos. Al finalizar el proceso de centrifugación se decantó el sobrenadante y se determinó el peso ganado por el gel a través de un cálculo matemático (ecuación 1). Esta metodología se basa en la establecida por Bryant \& Hamaker (1997) (citado en, Ocaña 2019).

$$
\text { CRA }(\%)=\frac{\text { Peso del gel }(\text { gramos })-\text { peso de muestra }(\text { gramos })}{\text { peso de muestra }(\text { gramos })}
$$

\section{Resultados.}

Los datos que se reportan fueron obtenidos sobre la base de la metodología indicada.

\section{Análisis proximal.}

Los resultados del análisis proximal de la harina de Mashua (Treopaeolum Tubersoum) en las variedades blanca, roja y negra se muestran en la Tabla 1. Los datos presentan desviaciones estándar cercanas a 0 por lo que se entiende que no existe dispersión entre los datos obtenidos. Cada una de las muestras se realizó por triplicado. El único parámetro que no se lo determinó en el laboratorio fue el extracto libre de nitrógeno (ELN), conformado por carbohidratos solubles en su mayoría, que fue obtenido por diferencia de 100 menos la suma de humedad, ceniza, grasa, fibra y proteína.

Tabla 1. Análisis proximal de harina de mashua (Treopaeolum Tubersoum)

\begin{tabular}{c|c|c}
\hline \multirow{2}{*}{ Parámetros } & Media & Desviación estándar \\
\cline { 2 - 3 } & $\overline{\boldsymbol{M}}$ & $\boldsymbol{\sigma}$ \\
\hline Humedad \% & $10,46 \pm 0,09$ & 0,085 \\
\hline Ceniza \% & $4,66 \pm 0,10$ & 0,095 \\
\hline Grasa \% & $0,81 \pm 0,24$ & 0,022 \\
\hline Fibra \% & $7,07 \pm 0,009$ & 0,008 \\
\hline Proteína \% & $12,34 \pm 0,08$ & 0,068 \\
\hline Carbohidratos \% & $64,67 \pm 0,14$ & 0,122 \\
\hline
\end{tabular}

Fuente: Elaboración propia.

Uno de los factores específicos que determina la calidad de la harina destinada a diferentes usos es el contenido de humedad. Los resultados mostrados en la Tabla 1 señalan que la humedad de harina de Mashua presenta un valor promedio de 10,46 $\pm 0,09$ $\%$, este valor se encuentra próximo al que establece la norma técnica INEN 616 (2015) para la Harina de trigo, que corresponde a un $14,5 \%$ para el contenido de máximo de humedad en harinas para todo uso (INEN,2015).

De acuerdo con lo mencionado por Santos (citado en Ocaña, 2019) y Lalaleo (2017) un bajo porcentaje de humedad tiene una relación directa con la calidad de la harina, ya que harinas que presentan un valor de humedad por debajo del $14 \%$ se pueden almacenar en temperatura ambiente sin ser susceptibles al ataque de hongos u otros microorganismos. 
Barbosa et al. (citado en Ocaña, 2019) concluye que el contenido de humedad corresponde a un factor primordial en la manipulación, almacenamiento y procesamiento de harinas. Así mismo, Guerra (2014) reporta un valor del 13,08\% de contenido de humedad para harina de mashua, valor similar al obtenido en la presente investigación para las tres variedades blanca, roja y negra. Por esta razón se puede manifestar que las muestras de harina de mashua analizadas presentan facilidad para el manejo y almacenamiento.

Del análisis proximal se puede determinar que el aporte de proteína para la harina de Mashua se encuentra en un rango de $12,34 \pm 0,08 \%$. Este valor promedio es superior al reportado por Salazar (2014) que determina un porcentaje de proteína en la harina de mashua correspondiente a $9.8 \%$ mayor al obtenido para la harina de trigo que fue de 8 al 14\%. (citado en Sarmiento, 2015)

La mashua posee elevada cantidad de aminoácidos esenciales como lisina, que es un aminoácido limitante en gran variedad de cereales y leguminosas (Espinoza et al., 2002). Según Espín et al (2004), la proteína encontrada en la harina de mashua presenta valores que van del 7,22 al 13,99\%, cantidades que están en dependencia de la variedad de la mashua siendo el valor obtenido un valor similar al reportado. (citado en Sarmiento,2015). De igual manera el contenido de proteína $12.90 \%$ de la harina de mashua es mayor al 7,0\% al referido en el documento del codex alimentarius (CODEX, 1985).

El contenido de cenizas para la harina de mashua presenta un valor promedio de $4,66 \pm 0,10 \%$. Con respecto al contenido de cenizas este valor coincide con el obtenido por Guerra (2014) que fue de 5,68\% en harina de mashua y Montero (2006) logró un valor similar en mashua fresca de 5,45\%. Según Samaniego (2010) la mashua es un producto rico en minerales como calcio y fósforo.

En el contenido de grasa presenta un valor promedio para la harina de mashua de $0,81 \pm 0,24 \%$. Según, Jitngarmkusol, Hongsuwankul, y Tananuwong (citados en Silva, 2019), manifiestan que la presencia de proteínas podría influir en determinación de contenido graso ya que los aminoácidos presentes en la estructura de las proteínas poseen cadenas laterales no polares que pueden interactuar de manera hidrófoba con las cadenas hidrocarbonadas de los lípidos.

Con respecto a la fibra según el análisis proximal las muestras analizadas presentan un

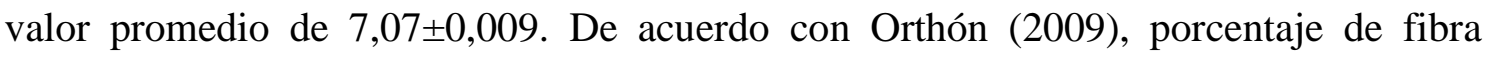
presente en harina de trigo es casi nula, debido a que durante el procesamiento se retiran elementos que contienen celulosa como son cubierta y germen. Las harinas integrales por su parte conservan el germen y la cubierta, razón por la cual la cantidad de fibra es mayor en relación a las harinas convencionales (citado en Guerra, 2014) 
Los resultados obtenidos para carbohidratos muestran valor promedio de $64,67 \pm 0,14 \%$. Estos valores se justifican con los resultados presentados por Bruin (2006) que establece que las harinas de panificación poseen un contenido de carbohidratos de 67 a $74 \%$. (Citado en Quintana,2015). De acuerdo con lo señalado por Coellar (2017) el potencial nutricional de la mashua está en su contenido de carbohidratos como fuente de energía. La presencia de glucosinatos en la mashua y su contenido de vitamina $\mathrm{C}$ determinarían que existe un elevado porcentaje de carbohidratos y por ende un potencial nutricional muy importante. (Romero de la Hoz, 2017)

Tabla 2. Análisis de varianza para los datos del análisis proximal

\begin{tabular}{|c|c|c|c|c|c|c|}
\hline \multicolumn{7}{|c|}{ HUMEDAD } \\
\hline $\begin{array}{c}\text { Origen de } \\
\text { las } \\
\text { variaciones }\end{array}$ & $\begin{array}{c}\text { Suma de } \\
\text { cuadrados }\end{array}$ & $\begin{array}{c}\text { Grados de } \\
\text { libertad }\end{array}$ & $\begin{array}{l}\text { Promedio } \\
\text { de los } \\
\text { cuadrados }\end{array}$ & $F$ & Probabilidad & $\begin{array}{c}\text { Valor } \\
\text { crítico para } \\
F\end{array}$ \\
\hline $\begin{array}{l}\text { Entre } \\
\text { grupos }\end{array}$ & 0,27555556 & 2 & 0,13777778 & 1,51219512 & 0,29390038 & 5,14325285 \\
\hline $\begin{array}{l}\text { Dentro de } \\
\text { los grupos }\end{array}$ & 0,54666667 & 6 & 0,09111111 & & & \\
\hline Total & 0,82222222 & 8 & & & & \\
\hline \multicolumn{7}{|c|}{ CENIZAS } \\
\hline $\begin{array}{c}\text { Origen de } \\
\text { las } \\
\text { variaciones }\end{array}$ & $\begin{array}{c}\text { Suma de } \\
\text { cuadrados }\end{array}$ & $\begin{array}{c}\text { Grados de } \\
\text { libertad }\end{array}$ & $\begin{array}{l}\text { Promedio } \\
\text { de los } \\
\text { cuadrados }\end{array}$ & $F$ & Probabilidad & $\begin{array}{c}\text { Valor } \\
\text { crítico para } \\
F\end{array}$ \\
\hline $\begin{array}{l}\text { Entre } \\
\text { grupos }\end{array}$ & 0,14408889 & 2 & 0,07204444 & 6,31353457 & 0,03342106 & 5,14325285 \\
\hline $\begin{array}{l}\text { Dentro de } \\
\text { los grupos }\end{array}$ & 0,06846667 & 6 & 0,01141111 & & & \\
\hline Total & 0,21255556 & 8 & & & & \\
\hline \multicolumn{7}{|c|}{ GRASA } \\
\hline $\begin{array}{c}\text { Origen de } \\
\text { las } \\
\text { variaciones }\end{array}$ & $\begin{array}{c}\text { Suma de } \\
\text { cuadrados }\end{array}$ & $\begin{array}{c}\text { Grados de } \\
\text { libertad }\end{array}$ & $\begin{array}{l}\text { Promedio } \\
\text { de los } \\
\text { cuadrados }\end{array}$ & $F$ & Probabilidad & $\begin{array}{c}\text { Valor } \\
\text { crítico para } \\
F \\
\end{array}$ \\
\hline $\begin{array}{l}\text { Entre } \\
\text { grupos }\end{array}$ & 0,00888889 & 2 & 0,00444444 & 5,12820513 & 0,05027821 & 5,14325285 \\
\hline $\begin{array}{l}\text { Dentro de } \\
\text { los grupos }\end{array}$ & 0,0052 & 6 & 0,00086667 & & & \\
\hline Total & 0,01408889 & 8 & & & & \\
\hline \multicolumn{7}{|c|}{ FIBRA } \\
\hline $\begin{array}{c}\text { Origen de } \\
\text { las } \\
\text { variaciones }\end{array}$ & $\begin{array}{c}\text { Suma de } \\
\text { cuadrados }\end{array}$ & $\begin{array}{c}\text { Grados de } \\
\text { libertad }\end{array}$ & $\begin{array}{l}\text { Promedio } \\
\text { de los } \\
\text { cuadrados }\end{array}$ & $F$ & Probabilidad & $\begin{array}{c}\text { Valor } \\
\text { crítico para } \\
F\end{array}$ \\
\hline $\begin{array}{l}\text { Entre } \\
\text { grupos }\end{array}$ & $6,6667 \mathrm{E}-05$ & 2 & $3,3333 \mathrm{E}-05$ & 0,15 & 0,8638376 & 5,14325285 \\
\hline $\begin{array}{l}\text { Dentro de } \\
\text { los grupos }\end{array}$ & 0,00133333 & 6 & 0,00022222 & & & \\
\hline Total & 0,0014 & 8 & & & & \\
\hline \multicolumn{7}{|c|}{ PROTEINA } \\
\hline $\begin{array}{c}\text { Origen de } \\
\text { las } \\
\text { variaciones }\end{array}$ & $\begin{array}{c}\text { Suma de } \\
\text { cuadrados }\end{array}$ & $\begin{array}{c}\text { Grados de } \\
\text { libertad }\end{array}$ & $\begin{array}{l}\text { Promedio } \\
\text { de los } \\
\text { cuadrados }\end{array}$ & $F$ & Probabilidad & $\begin{array}{c}\text { Valor } \\
\text { crítico para } \\
F \\
\end{array}$ \\
\hline
\end{tabular}




\begin{tabular}{c|c|c|c|c|c|c}
\hline $\begin{array}{c}\text { Entre } \\
\text { grupos }\end{array}$ & 0,06486667 & 2 & 0,03243333 & 1,90162866 & 0,22926753 & 5,14325285 \\
\hline $\begin{array}{c}\text { Dentro de } \\
\text { los grupos }\end{array}$ & 0,10233333 & 6 & 0,01705556 & & & \\
\hline Total & 0,1672 & 8 & & & & \\
\hline
\end{tabular}

Fuente: Elaboración propia.

$\mathrm{Al}$ realizar el análisis de varianza de las muestras tomadas con tres repeticiones y con un valor de $\alpha=0,05$, se encontró que no existe diferencia altamente significativa entre cada una de las muestras analizadas. Lo que se demuestra que las muestras tienen similitud en el porcentaje de humedad. Una vez realizado los ANOVA de los cinco parámetros analizados para cada muestra de harina de mashua, se puede observar de acuerdo a los resultados obtenidos que en la totalidad de los casos se acepta hipótesis nula es decir que las medias de los datos obtenidos no presentan diferencias significativas, es decir con un nivel de confianza del $95 \%$, se observa que la colección de datos obtenidos de las muestras de harina de mashua son similares y concordantes.

\section{Determinación de Acidez titulable y pH.}

La tabla 4, muestra los valores obtenidos para el parámetro $\mathrm{pH}$. De acuerdo con los

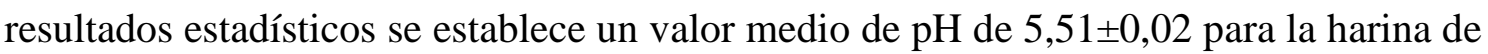
Mashua. Según Bennion (citado en Sarmiento,2015), la capacidad del gluten para formar la red esponjosa está influida por el pH de la masa formada por la harina y la actividad proteolítica de la enzima.

Tabla 3. Resultados de medición $\mathrm{pH}$ para harina de mashua (Tropaleum tuberosum)

\begin{tabular}{|c|c|c|c|c|c|c|}
\hline \multirow{4}{*}{$\begin{array}{c}\text { pH de Harina de } \\
\text { mashua }\end{array}$} & M1 & M2 & M3 & $\bar{M}$ & $\boldsymbol{\sigma}$ & Margen de error \\
\hline & 5,48 & 5,51 & 5,48 & \multirow{3}{*}{5,513} & \multirow{3}{*}{0,018} & \multirow{3}{*}{0,020} \\
\hline & 5,53 & 5,53 & 5,51 & & & \\
\hline & 5,52 & 5,53 & 5,53 & & & \\
\hline
\end{tabular}

Fuente: Elaboración propia.

En la tabla 5 se muestran los resultados obtenidos para el análisis de acidez titulable de

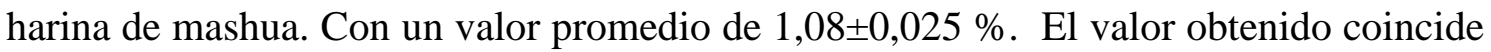
con lo reportado por Saa (2019), que establece valores de 1,15 a 1,38\% de acidez expresada como porcentaje ácido tartárico para muestras de harina de mashua. De acuerdo con Aguado (2017) estos valores podrían corresponder a la composición del tubérculo que presenta ácidos oxálico y ascórbico, cuyo porcentaje según Aguado (2017) puede variar de acuerdo con el tipo de cultivo y variedad. (citado en Ocaña, 2019) 
Tabla 4. Resultados para la determinación de porcentaje de acidez titulable en harina de mashua (Tropaleum tuberosum)

\begin{tabular}{c|r|r|r|c|c|c}
\hline \multirow{2}{*}{$\begin{array}{c}\text { \%acidez } \\
\text { (ácido }\end{array}$} & M1 & M2 & M3 & $\overline{\boldsymbol{M}}$ & $\boldsymbol{\sigma}$ & Margen de error \\
\cline { 2 - 5 } tartárico) & 1,08 & 1,13 & 1,09 & & & \\
\cline { 2 - 5 } & 1,05 & 1,08 & 1,07 & \multirow{2}{*}{1,086} & 0,023 & 0,025 \\
\cline { 2 - 4 } & 1,06 & 1,12 & 1,09 & & & \\
\hline
\end{tabular}

Fuente: Elaboración propia.

De igual manera se puede establecer que existe un porcentaje mayor de acidez titulable comparada con la harina de trigo ya que en los requisitos de la norma NTE INEN 616: 98 Requisitos para la harina de trigo, se establece un máximo de acidez $0,1 \%$ (en porcentaje de masa de ácido sulfúrico). (INEN, 2015).

\section{Determinación de Actividad de agua (aw).}

En la tabla 6. Se muestran los valores de actividad de agua de las muestras de harina de mashua evaluadas, con un valor promedio de 0,36 $\pm 0,01$. Este valor de actividad de agua (aw) es inferior al reportado por Arellano y Zavaleta (2017) para la harina de trigo que fue de $0.58 \pm 0.011$.

Tabla 5. Resultados de la determinación de actividad de agua para harina de mashua (Tropaleum tuberosum)

\begin{tabular}{c|c|c|c|c|c|c}
\hline \multirow{4}{*}{$\begin{array}{c}\text { Actividad } \\
\text { de agua aw }\end{array}$} & \multicolumn{1}{|c|}{ M1 } & M2 & M3 & $\overline{\boldsymbol{M}}$ & $\boldsymbol{\sigma}$ & Margen de error \\
\cline { 2 - 5 } & 0,36 & 0,38 & 0,36 & & & \\
\cline { 2 - 4 } & 0,36 & 0,38 & 0,35 & 0,364444444 & 0,00949334 & 0,010742723 \\
\cline { 2 - 4 } & 0,35 & 0,38 & 0,36 & & & \\
\hline
\end{tabular}

Fuente: Elaboración propia.

De acuerdo con la investigación de Montoya y Giraldo (2010) se presentan un valor de 0.651 para la harina de trigo que de igual manera es valor superior al valor reportado para la harina de mashua en la presente investigación. Mientras más alta sea la aw y más se acerque a 1.0 mayor será su inestabilidad por lo cual se debe de tener un valo entre 0,6 a 0,7 para retardar el crecimiento microbiano (Badui, 2010), considerando que la actividad de agua define su disponibilidad para reacciones químicas y desarrollo microbiano. (citado en Lara et al, 2017)

\section{Capacidad de retención de agua \% CRA.}

Los resultados de la determinación de la capacidad de absorción de agua CRA (\%) se muestran en la tabla 7, donde se pueden evidenciar variaciones entre 5,05 a una temperatura de $50^{\circ} \mathrm{C}$ y 8,63 a una temperatura de $80^{\circ} \mathrm{C}$ para las muestras de harina de 
mashua utilizadas. Estos valores son concordantes con los expuestos por Ocaña (2019), quien determina un valor de capacidad de retención de agua para la harina de mashua de $8,60 \%$ a $80^{\circ} \mathrm{C}$. Los datos obtenidos para las muestras de mashua presentan los valores elevados con respecto a la harina de trigo que según la investigación de Sandoval et al (2012) fue de 1,92 $\pm 0,06$, esto puede deberse a la composición química de la harina de mashua sobre todo por su contenido de carbohidratos, fibra dietética, interacción entre agua - proteína y el contenido de almidón según lo expresado por Traynhan et al (2013) (citado en Ocaña, 2017).

Tabla 6. Resultados promedio de la capacidad de retención de agua de harina de mashua a diferentes temperaturas.

\begin{tabular}{c|c|c|c|c}
\hline \multirow{2}{*}{ MUESTRA } & \multicolumn{4}{|c}{ CRA (\%) } \\
\cline { 2 - 5 } & $\mathbf{5 0}^{\circ} \mathbf{C}$ & $\mathbf{6 0}^{\circ} \mathbf{C}$ & $\mathbf{7 0}^{\circ} \mathbf{C}$ & $\mathbf{8 0}^{\circ} \mathbf{C}$ \\
\hline Harina de mashua & $5,05 \pm 0,056$ & $7,16 \pm 0,14$ & $8,33 \pm 0,10$ & $8,63 \pm 0,0533$ \\
\hline
\end{tabular}

Fuente: Elaboración propia.

Según Ram, Dawar, Singh y Shoran (2005) en su investigación observaron harinas con valores altos de CRA $(5,3$ a 7,6\%) y fuerza de gluten intermedia, mencionan que este tipo de harinas también que harinas son usadas preferentemente en la elaboración de pan sin levadura. (citado en Vásquez et al, 2017). Uno de los aspectos determinantes para la capacidad de retención de agua es el tamaño de partícula. Para Berton et al (2002) el incremento en almidón dañado puede influir sobre el aumento en la capacidad de retención de agua. Cuando la temperatura se incrementa, se produce una limitación del gránulo del almidón al hinchamiento durante la hidratación, en condiciones de agitación y calentamiento bajo las cuales se desarrolla la prueba de CRA, se ocasiona un incremento en el número de puentes disulfuro de las proteínas y por lo tanto la pérdida de solubilidad, lo que explicaría que el almidón retarde su hinchamiento del gránulo. (citado en Vásquez, 2017)

En la Tabla 8 se muestran los resultados del análisis estadístico realizado ADEVA de dos factores, demostrando que existen diferencias significativas ( $p>0,05)$ para los valores de capacidad de retención de agua para las muestras de harina de mashua sometidas a cuatro temperaturas diferentes $50,60,70$ y $80^{\circ} \mathrm{C}$.

Tabla 7. Análisis de varianza para los datos de capacidad de retención de agua en muestras de harina de mashua (Tropoleum tuberosum)

\begin{tabular}{ccccccc}
\hline $\begin{array}{c}\text { Origen de } \\
\text { las } \\
\text { variaciones }\end{array}$ & $\begin{array}{c}\text { Suma de } \\
\text { cuadrados }\end{array}$ & $\begin{array}{c}\text { Grados de } \\
\text { libertad }\end{array}$ & $\begin{array}{c}\text { Promedio } \\
\text { de los } \\
\text { cuadrados }\end{array}$ & F & Probabilidad & $\begin{array}{c}\text { Valor } \\
\text { crítico } \\
\text { para F }\end{array}$ \\
\hline temperaturas & 23,6875583 & 3 & 7,89585278 & 622,401358 & $7,18 \times 10^{-08}$ & 4,75706266 \\
muestras & 0,01115 & 2 & 0,005575 & 0,43945697 & 0,663581273 & 5,14325285
\end{tabular}


Error $\quad 0,07611667 \quad 6 \quad 0,01268611$

Total $\quad 23,774825 \quad 11$

Fuente: Elaboración propia.

El valor de la capacidad de retención de agua CRA a temperaturas menores de $70^{\circ} \mathrm{C}$ indica que los gránulos se resistieron al hinchamiento y la absorción de agua fue menor. Entre los 70 y 80 grados Celsius, los gránulos experimentan un hinchamiento gradual, siendo mayor a $80^{\circ} \mathrm{C}$, según Rao y Tattiyakul (1999) pudiendo deberse a la ruptura de los puentes de hidrógeno intramoleculares que le permiten una absorción irreversible y progresiva del agua (citado en Miranda, 2012)

\section{Conclusiones:}

- El análisis proximal realizado a la harina de mashua determina la presencia de un porcentaje importante de proteína correspondiente a $12,34 \%$ y de igual manera un contenido favorable de fibra $7,07 \%$, estos resultados evidencian su calidad nutricional, funcionalidad reológica y establecen sus posibles aplicaciones.

- El porcentaje de acidez titulable obtenido para la harina de mashua mostró valores elevados $(1,08 \%)$ que se pueden atribuir al importante contenido de ácido ascórbico y acido oxálico.

- El porcentaje de capacidad de retención de agua $(5,0,5 \%)$ para la harina de mashua fue superior al reportado para harinas como la de trigo, atribuyéndose este resultado a su importante contenido de carbohidratos y fibra dietética, además de su capacidad higroscópica por su elevado nivel proteína.

- Los altos valores obtenidos para la determinación de la capacidad de retención de agua para las muestras de harina de mashua, hacen posible considerarla para ser incorporada en la elaboración de productos cárnicos (embutidos) y algunos productos de panificación para proporcionarles su textura característica.

- La capacidad de retención de agua mostró un incremento conforme la temperatura del ensayo siendo de $5,05 \%$ en $50^{\circ} \mathrm{C}$ y $8,63 \%$ a los $80^{\circ} \mathrm{C}$ presentando diferencias estadísticamente significativas $(\mathrm{p}<0,05)$ entre temperaturas. Estos valores de CRA se asocian a una mayor cantidad de amilosa que se traduce en una mejor dispersión en soluciones acuosas y mejora en la retención de agua para las preparaciones alimenticias a la que se incorpore esta harina. 


\section{Referencias bibliográficas:}

Arbizu, C. y. (1993). Aproximación Agroecosistémica. El Agroecosistema Andino: problemas, limitaciones, perspectivas. Anales del Taller Internacional sobre el Agroecosistema Andino. Lima: CIP.

Arellano, E y Zavaleta, I. (2017). "Efecto de la sustitución parcial de harina de trigo (triticum aestivum) por harina de arvejas (pisum sativum) y harina de camote (ipomoea batatas) en las características tecnológicas y sensoriales de cupcake" (Universidad Nacional del Santa). Recuperado de: http://repositorio.uns.edu.pe/handle/UNS/2748

Association Of Official Analytical Chemists. Official Methods of Analysis of AOAC. 16th ed. USA, Washington, 1995.

Badui, S. D. 2010. Química de lós Alimentos. V. Pearson. D.F. México. México. 648 pag Bernabé, Y y Cancho, F. (2017). "Caracterización fisicoquímica, fitoquímica y funcional de la harina de khaya y oca" (Universidad Nacional del Centro del Perú Huancayo) Recuperado

de: http://repositorio.uncp.edu.pe/bitstream/handle/UNCP/1221/TESIS\%20YESICA $\%$ 20BERNAB\%c3\%89.pdf? sequence $=3 \&$ is Allowed $=\mathrm{y}$

Campos, D., Noratto, D., Chirinos, R., Arbizu, C., Roca, W., \& Zevallos, y. C. (2006). Antioxidant capacity and secondary metabolites in tour species of Andean tuber crops: Native potato (Solanum sp.), mashua. En R. e. Pavón, Native potato (Solanum sp.), mashua (pág. 86). Lima: Journal of the Science of Food and Agriculture.

Codex. (1985). Harina de Trigo. (Codex Standard 152). http://www.fao.org/input/download/standards/50/CXS_152s.pdf

Guerra, A. (2014). "Estudio de la utilización de la harina de mashua (Tropaeolum tuberosum) en la obtención del pan de molde" (Universidad tecnológica equinoccial).

Recuperado

de http://repositorio.ute.edu.ec/bitstream/123456789/5061/1/55524_1.pdf

Guerra, A. (2014). "Estudio de la utilización de la harina de mashua (Tropaeolum tuberosum) en la obtención del pan de molde" (Universidad tecnológica equinoccial). Recuperado de http://repositorio.ute.edu.ec/bitstream/123456789/5061/1/55524_1.pdf

INEN (1980). Harinas de origen vegetal. Determinación de proteína (NTE-INEN 519). https://www.normalizacion.gob.ec/buzon/normas/519.pdf

INEN (1980). Harinas de origen vegetal. Determinación de pérdida por calentamiento (NTE-INEN 518). https://www.normalizacion.gob.ec/buzon/normas/518.pdf

INEN (1980). Harinas de origen vegetal. Determinación de cenizas (NTE-INEN 520). https://www.normalizacion.gob.ec/buzon/normas/520.pdf

INEN (2012). Harinas de origen vegetal. Determinación de la fibra cruda. (NTE-INEN 522-2012). https://181.112.149.204/buzon/normas/nte_inen_522-1.pdf 
INEN (2013). Harinas de origen vegetal. Determinación de la Acidez titulable. (NTEINEN 521:2013). https://181.112.149.204/buzon/normas/521-1R.pdf

INEN (2013). Harinas de origen vegetal. Determinación de la concentración de Ión $\begin{array}{lllll}\text { hidrógeno } & \mathrm{o} & \mathrm{pH} & \end{array}$ https://www.normalizacion.gob.ec/buzon/normas/526-1R.pdf

INEN. (2015). Harina de trigo. Requisitos (NTE INEN.616). https://181.112.149.204/buzon/normas/nte-inen-616-4.pdf

Lalaleo, D. (2017). "Caracterización reológica de suspensiones elaboradas a partir harina y residuos de banano de rechazo" (Universidad Técnica de Ambato. Facultad de Ciencia e Ingeniería en Alimentos. Carrera de Ingeniería en Alimentos.). Recuperado de http://repositorio.uta.edu.ec/handle/123456789/24739

Lara, F. et al. (2017) Efecto de la sustitución de harina de trigo con harina de avena, maíz y sorgo sobre las propiedades reológicas de la masa, texturales y sensoriales del pan

Miranda, P. (2012). Caracterización Funcional del Almidón de Fríjol Zaragoza (Phaseolus Lunatus L.) y Cuantificación de su Almidón Resistente. Scielo.org.co. Retrieved 2 July 2020, from http://www.scielo.org.co/pdf/teclo/n30/n30a02.pdf.

Ocaña, I. (2019). “Caracterización Fisicoquímica, Nutricional y Reológica De Cultivos Andinos Infrautilizados" (Universidad Técnica de Ambato) Recuperado de https://repositorio.uta.edu.ec/bitstream/123456789/30002/1/AL\%20707.pdf

Quintana, T. (2015). "Estudio de la sustitución parcial de harina de Trigo con harina de quinua cruda y tostada en la Elaboración de pan" (Universidad tecnológica equinoccial). Recuperado de: http://repositorio.ute.edu.ec/bitstream/123456789/14405/1/61827_1.pdf

Romero de la Hoz, D. et al (2017). "Caracterización fisicoquímica, funcional, reológica y composicional de la harina precocida de cubio (Tropaeolum tuberosum R\&P) cultivado en diferentes fuentes de fertilización". (Universidad de la Salle). Recuperado de: https://ciencia.lasalle.edu.co/cgi/viewcontent.cgi?article=1073\&context=ing_ali mentos

Saa, M. (2019). Evaluación del efecto de secado de la mashua morada tropaeolum tuberosum sobre las propiedades organolépticas y actividad antioxidante (Universidad Técnica del Norte). Retrieved from http://repositorio.utn.edu.ec/handle/123456789/9033

Samaniego, L. (2010). "Caracterización de la mashua (tropaeolum tuberosum c) en el Ecuador”. (Universidad Tecnológica Equinoccial). Recuperado de http://repositorio.ute.edu.ec/bitstream/123456789/4870/1/41732_1.pdf

Sarmiento, Y. (2015). "Estudio de la sustitución parcial de la harina de Trigo por la harina de amaranto crudo y tostado en la elaboración de pan" (Universidad tecnológica equinoccial). Recuperado de: http://repositorio.ute.edu.ec/bitstream/123456789/5131/1/59116_1.pdf 


\section{PARA CITAR EL ARTÍCULO INDEXADO.}

González Cabrera, M. V., Moreno Andrade, G. I., \& López Sampedro, S. E. (2020). Caracterización nutricional y funcional de la harina de mashua. ConcienciaDigital, 3(3), 199-214. https://doi.org/10.33262/concienciadigital.v3i3.1299

\section{Liencia}

El artículo que se publica es de exclusiva responsabilidad de los autores y no necesariamente reflejan el pensamiento de la Revista Conciencia Digital.

El artículo queda en propiedad de la revista y, por tanto, su publicación parcial y/o total en otro medio tiene que ser autorizado por el director de la Revista Conciencia Digital.

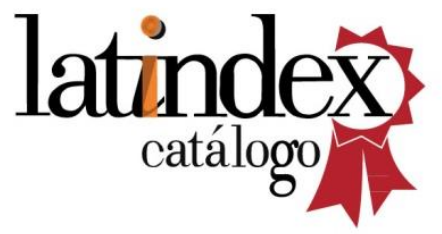

